\title{
A tecnologia digital como uma ferramenta de aprendizagem nas aulas de Matemática: criação de aplicativos para estudo do Teorema de Pitágoras.
}

\section{Digital technology as a learning tool in Mathematics class: creating apps to study the Pythagorean Theorem}

\author{
Adriana Dada de Andrade (adriandrade144@gmail.com) \\ Escola Estadual de Educação Básica Prudente de Morais, Osório \\ Universidade Federal do Rio Grande - Campus Santo Antônio da Patrulha (FURG / PPGECE)
}

Gilmar de Evangelho Pereira (gilmarevangelho@gmail.com)

Escola Estadual de Educação Básica Prudente de Morais, Osório

Resumo: O presente relato de experiência descreve atividades desenvolvidas com a turma Totalidade 8 da modalidade de Jovens e Adultos (EJA) de uma escola da rede pública estadual no município de Osório/RS, a maioria dos estudantes dessa turma eram jovens egressos do ensino regular, que devido as inúmeras evasões e repetências estavam fora da idade-série, tendo como oportunidade para concluírem seus estudos a EJA. Como uma alternativa diferente do ensino tradicional para abordar o Teorema de Pitágoras, surgiu a ideia de propor aos estudantes a criação de aplicativos relacionados ao assunto nas aulas de Matemática. Foi usada a plataforma App Inventor, que possibilita o desenvolvimento de aplicativos de maneira intuitiva utilizando o computador e a instalação desses aplicativos nos smartphones dos estudantes no final do projeto. Constatou-se que as atividades desenvolvidas contribuíram para auxiliar o estudante na construção do seu conhecimento de forma autônoma, despertaram a motivação e o interesse dos mesmos durante as aulas de Matemática. Assim, a proposta de criação de aplicativos apresentou-se como uma excelente alternativa para utilização no ensino dos conteúdos de Matemática.

Palavras-chave: Aplicativos; Ensino de Matemática; App Inventor.

Abstract: This experience report describes the activities developed with the class "Totalidade 8" of the "Ensino de Jovens e Adultos" (EJA) of a state public school in the city of Osório/RS; most students of this class were young adults secluded from regular education, because of evasions and failing classes they were not able to attend the right age-grade and were given the opportunity to finish their studies with EJA program. As a different alternative from the traditional approach of the Pythagorean Theorem, it was proposed to the students the idea of creating apps related to the subject in the Mathematics class. The App Inventor platform was used because it allows the development of apps on an intuitive way using the computer and it also allows the installation of these apps on the student's smartphones by the end of the project. The activities developed contributed to assist the students on their knowledge construction autonomously, helped them with motivation and their interest during the class. This

Recebido em: 30 /04/ 2020

Aceito em: $19 / 11 / 2020$ 
Edição Especial: XVI Encontro sobre Investigação na Escola - EIE

way, the proposal to create the apps was an excellent alternative to use in the teaching of Mathematical subjects.

Keywords: Apps; Teaching of Mathematics; App Inventor.

\section{INTRODUÇÃO}

Alguns dos desafios que se apresentam atualmente são desenvolver formas para tornar as aulas de Matemática mais atraentes, aproximar os conteúdos abordados do cotidiano dos estudantes e, ao mesmo tempo, propor situações que contribuam para o protagonismo desses nos processos de ensino e aprendizagem.

A preocupação dos professores de Matemática com o baixo desempenho dos alunos e a pouca motivação dos mesmos é recorrente nas reuniões pedagógicas das escolas e encontros de formação. As avaliações externas também apontam o baixo desempenho dos estudantes brasileiros em Matemática. Dados obtidos a partir do Programme for International Student Assessment (Pisa) de 2018, divulgados pelo Instituto Nacional de Estudos e Pesquisas Educacionais Anísio Teixeira (Inep), vinculado ao Ministério da Educação (MEC), ao comparar o desempenho dos nossos estudantes em Matemática com o desempenho de estudantes de outros países da América do Sul, também analisados pelo Pisa, conclui que o Brasil possui um dos piores resultados. Além disso, os resultados do Sistema de Avaliação da Educação Básica (Saeb) revelam que uma parte considerável dos estudantes apresentaram níveis insuficientes de proficiência em Matemática.

Diante desse contexto, uma das alternativas encontradas para motivar a participação do estudante, aproximar o conteúdo do seu cotidiano e melhorar seu desempenho foi propor a criação de aplicativos para o estudo do Teorema de Pitágoras.

A atividade foi realizada com a turma T8 da modalidade Ensino de Jovens e Adultos (EJA) de uma escola da rede pública estadual, turma essa escolhida por apresentar características distintas, quase a totalidade dos alunos matriculados eram adolescentes com histórico de reprovações e evasões que ao completar 18 anos de idade no Ensino Médio migraram para a EJA.

Faz parte desse relato o detalhamento das atividades que foram desenvolvidas, a apresentação de uma amostra da pesquisa de opinião dos estudantes sobre as atividades 
Edição Especial: XVI Encontro sobre Investigação na Escola - EIE

e também a análise e discussão do relato. Nas considerações finais, apresenta-se uma reflexão sobre a experiência e sua contribuição nos processos ensino e aprendizagem.

\section{CONTEXTO E DETALHAMENTO DAS ATIVIDADES:}

A tecnologia digital está a cada dia mais presente na sociedade, um exemplo disso é o smartphone, que está integrado a vida das pessoas, os aplicativos instalados nesses dispositivos móveis apresentam muitas possibilidades de uso nas mais diversas áreas. A maioria dos estudantes que frequentam a escola está sempre fazendo uso de seus smartphones, utilizando com muita habilidade aplicativos de comunicação, de jogos, de mídia, entre outros. Para Brackmann et al. (2017, p. 982-991) : “nos tempos atuais, o desafio que se impõe aos usuários é criar seus próprios sistemas (por exemplo, programas e jogos) ou modificar os existentes de acordo com sua necessidade pessoal".

Nesse sentido, foi criadas atividades utilizando uma plataforma on-line gratuita, de fácil acesso, em português, denominada App Inventor. Ela permite a criação de aplicativos no computador para depois instalá-los nos smartphones, assim definida:

[...] é uma plataforma de desenvolvimento, que permite pessoas com qualquer nível de experiência em programação criarem programas (aplicações) para o sistema operacional Android. Ele usa uma interface gráfica onde a funcionalidade dos componentes é exposta aos desenvolvedores via blocos de código permitindo construir o aplicativo sem ter que escrever código tradicional, tal como montar um quebra-cabeça (BARBOSA, 2016, p.26).

A utilização dessa plataforma coloca a disposição do jovem a possibilidade de ser um produtor de tecnologia, deixando de ser apenas um consumidor da mesma. Essa capacidade é essencial nos dias atuais, uma vez que vivemos em uma sociedade que está sempre em transformação, exigindo novas competências e habilidades para enfrentar o mercado de trabalho.

A atividade proposta está em conformidade com a Base Nacional Curricular Comum (BNCC) no que diz respeito à utilização das experiências presentes no dia a dia dos estudantes como um ponto de partida para produzir novos conhecimentos e ao papel fundamental do uso da tecnologia nesse contexto. 
Assim, essas atividades tiveram como objetivo a criação de um aplicativo para o smartphone com o propósito de facilitar aprendizagem do Teorema de Pitágoras, buscando despertar o interesse do estudante nos processos ensino e aprendizagem e tornando o estudante o protagonista.

As atividades foram desenvolvidas com a turma T8 da modalidade EJA (correspondente ao segundo ano do Ensino Médio), no laboratório de informática da escola, durante as aulas de Matemática. Para isso, foi usada a plataforma App Inventor, os aplicativos foram desenvolvido por duplas de estudantes e com a mediação do professor no período de 17 a 24 de janeiro de 2020, perfazendo 8 períodos.

No primeiro momento, com a utilização do data show, foi apresentado aos estudantes o App Inventor, sendo constatado que nenhum deles conhecia a plataforma. Após uma explanação sobre principais recursos do App Inventor, os estudantes acessaram a plataforma utilizando sua conta do Google, a partir de então, eles puderam verificar na prática os recursos do App Inventor, detalhados na explanação inicial, ou seja, as duas janelas, Designer e Blocos. A primeira para realizar a interface do aplicativo e a segunda para a programação, realizada por meio de blocos encaixáveis com as funções de lógica, matemática, texto, cores, variáveis, procedimentos, entre outros, que se encaixam para executar comandos. Também foi mostrado aos estudantes como salvar imagens para utilização no aplicativo.

Em seguida, foi proposto o desafio para que, em duplas, criassem um aplicativo capaz de calcular a medida da hipotenusa e dos catetos de triângulos retângulos usando o Teorema de Pitágoras. Inicialmente, os estudantes pensaram no designer do aplicativo, salvaram as imagens para utilização, assim usando sua criatividade e autonomia, cada dupla criou um designer personalizado para seu projeto.

No momento seguinte, iniciou-se a parte da programação, essa sem dúvida, foi a parte da atividade que exigiu uma atenção especial, algumas duplas precisaram do auxilio do professor na execução. Numa primeira etapa os estudantes desenvolveram a programação para calcular a hipotenusa, encaixaram os blocos e examinaram a funcionalidade do aplicativo no seu smartphone, as duplas cujos aplicativos não funcionaram corretamente foram orientadas a localizar o erro, antes de ampliar o projeto com a utilização de mais blocos. 
Edição Especial: XVI Encontro sobre Investigação na Escola - EIE

ISSN: 2595-4520 Vol. 4, n. 2. 2021

Quando os estudantes verificaram que os projetos estavam funcionando corretamente, os aplicativos foram compilados e instalados nos smartphones e utilizados na resolução de alguns exercícios de aplicação do Teorema de Pitágoras, como uma forma de constatar a eficiência do aplicativo por eles desenvolvido.

Ao término das atividades os estudantes responderam uma pesquisa onde puderam expressar sua opinião sobre as mesmas.

\section{Avaliação dos alunos sobre as contribuições das atividades com 0 App Inventor para seu aprendizado} $\mathrm{N}^{\circ}$ de estudantes

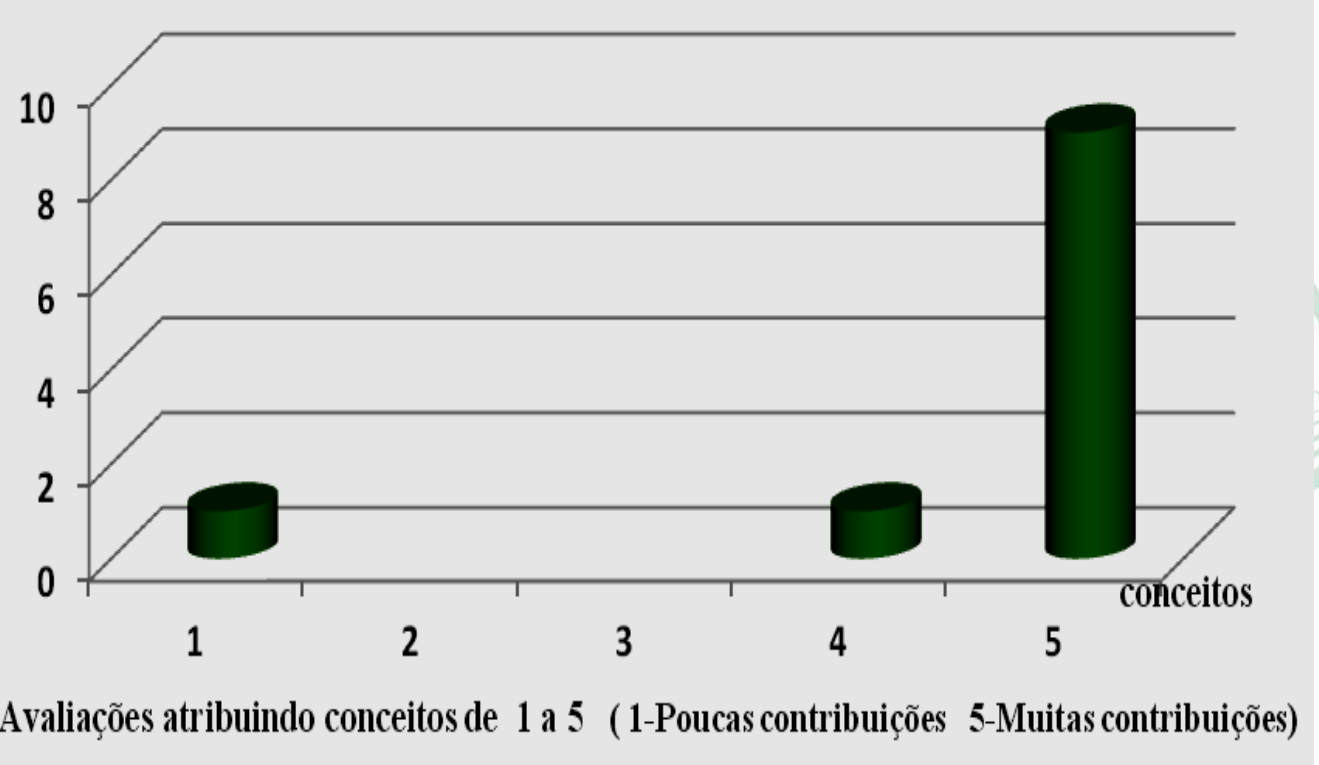

Figura 1 - Respostas dos alunos no questionário de avaliação das atividades. 


\section{Opinião dos alunos a respeito do uso do smartphone na sala de aula}

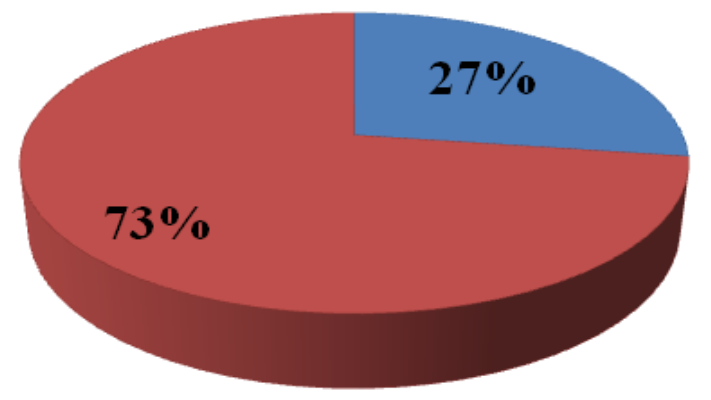

$\square$ Uso livre (Inclusive redes sociais e jogos diversos)

$\square$ Uso apenas para as atividades escolares

Figura 2 - Respostas quando questionados ao uso do smartphone em aula.

Respostas dos alunos em uma das questões do formulário de avaliação:

Qual a sensação de ter um App de sua autoria instalado no smartphone?

Estudante "A": Foi uma sensação boa, bem gratificante em saber que eu consegui fazer um App".

Estudante "B": "Uma sensação totalmente inovadora para as aulas"

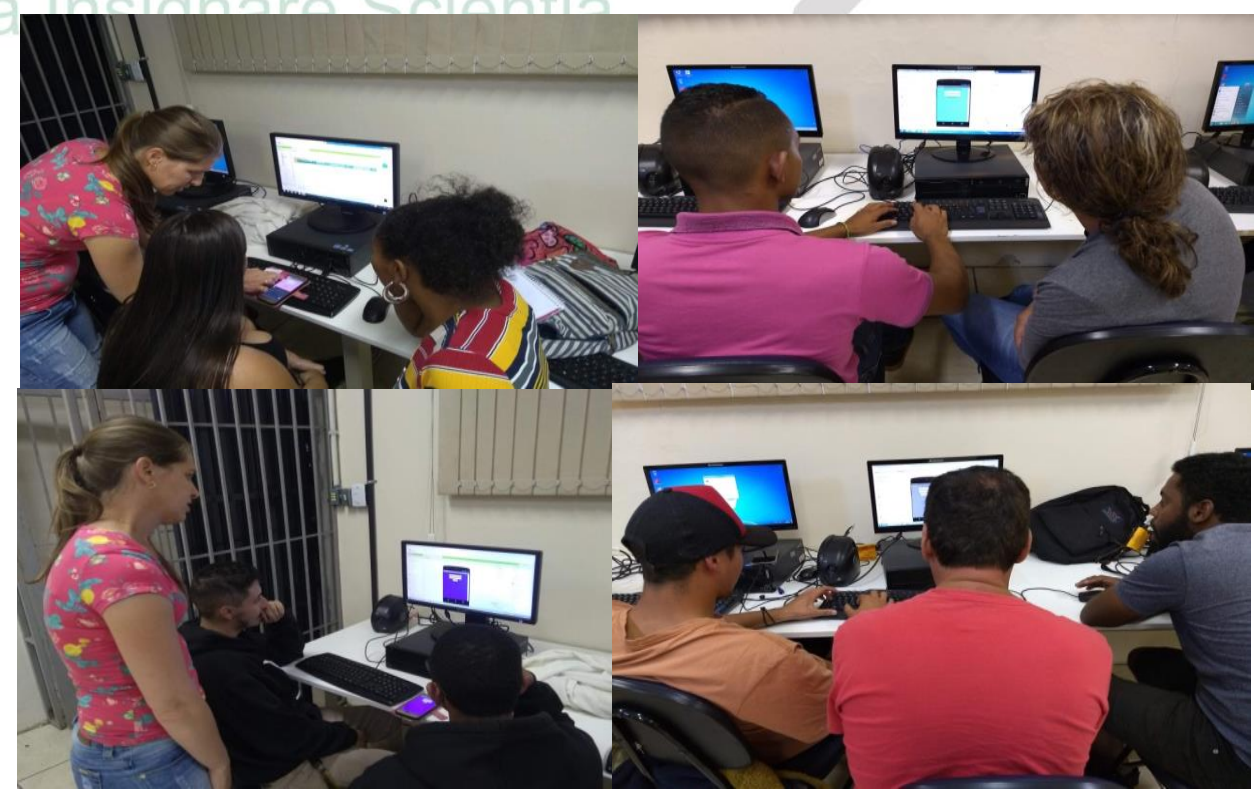

Figura 3 - Estudantes no laboratório de informática criando seus aplicativos 


\section{ANÁLISE E DISCUSSÃO DE RELATO}

No desenvolvimento das atividades utilizando o App Inventor, foi observado que os estudantes ficaram muito animados em criar um aplicativo para o seu smartphone. Por meio do questionário on-line da plataforma Google, preenchido pelos estudantes, concluiu-se que eles acharam muito interessantes as atividades desenvolvidas.

Observou-se que a ferramenta App Inventor incentivou a criatividade, curiosidade e autonomia dos estudantes, que segundo Paulo Freire (1996), devem ser respeitadas e estimuladas pelo educador na prática educativa, rejeitando assim a educação "bancária", em que o estudante é um ser passivo, apenas recebe o conteúdo e o professor é visto como o "dono" do conhecimento.

O ambiente do laboratório contribuiu para uma aula onde o professor e os estudantes aprenderam juntos, de acordo com Krause, Felber e Venquiaruto (2018, p.7):

As inovações tecnológicas possibilitaram o surgimento de uma nova sala de aula e a mudança do papel docente, que passa a ser um guia dos alunos em meio à Sociedade da Informação, fazendo com que as aulas ocorram de forma mais dinâmica com maior interação entre professor aluno, onde o conhecimento não é apenas transmitido, mas construído.

O estudante construiu seu conhecimento de maneira prazerosa e por meio da programação desenvolveu o pensamento computacional, que segundo Wing (2016) é uma habilidade fundamental para todos, envolve a criação de estratégias para a resolução de um problema, o planejamento, a tomada de decisões, a pesquisa.

Além disso, a atividade apresentada convergiu com a BNCC (Base Nacional Comum Curricular), documento de caráter normativo, que apresenta o desenvolvimento da Cultura Digital como uma das competências a serem desenvolvidas nos estudantes.

\section{CONSIDERAÇÕES FINAIS}

O projeto usando o App Inventor para criação de um aplicativo associado ao Teorema de Pitágoras surgiu como uma alternativa diferente daquelas normalmente utilizadas. Desde sua idealização prevíamos que alguns obstáculos surgiriam, como por exemplo, o fato dos estudantes usarem predominantemente seus smarphones para acesso às redes sociais, não lembrarem seu próprio e-mail (que é necessário para acesso

Recebido em: $30 / 04 / 2020$ 
Edição Especial: XVI Encontro sobre Investigação na Escola - EIE

à plataforma), a pouca persistência para solucionar os erros cometidos durante a execução da atividade, a falta de pré-requisitos dos conteúdos de Matemática.

No entanto, no desenvolvimento das atividades, os estudantes mostraram-se muito receptivos, com muita vontade e interesse em aprender, inclusive manifestaram a intenção de criar outros aplicativos com o App Inventor e fizeram muitos questionamentos a respeito da plataforma. Foram aulas muito interessantes, em que os estudantes construíram o seu conhecimento, tiveram a oportunidade de aprender com seu erros, pois quando o aplicativo era testado no smartphone e não funcionava como deveria, os estudantes descobriram os erros cometidos e buscavam corrigi-los para que o processo fosse concluído com sucesso.

O smartphone aliado ao App Inventor foi uma ferramenta que auxiliou os estudantes na construção do seu conhecimento. Essa atividade, além de trabalhar o Teorema de Pitágoras desenvolveu o pensamento computacional, a criatividade e a autonomia. Um aspecto importante da atividade a ser considerado foi o contato dos estudantes com a plataforma, assim puderam conhecer como os aplicativos usados em seus smartphones podem ser desenvolvidos, sendo um ponto de partida, para que tenham condições de desenvolver seus próprios aplicativos de acordo com seus interesses e necessidades.

A experiência foi gratificante, pois envolveu os estudantes nas atividades, despertando o interesse e a motivação dos mesmos. Pelos motivos apresentados, pretende-se realizar mais projetos usando o App Inventor nas aulas de Matemática.

\section{REFERÊNCIAS}

BARBOSA, Marcos Alberto. Desenvolvendo Aplicativos Para Dispositivos Móveis através do MIT App Inventor 2 nas Aulas de Matemática. 2016. 142 f. Dissertação (Mestrado Profissional em Matemática em Rede Nacional). Universidade Estadual de Santa Cruz. 2016. Disponível em: https://sca.profmatsbm.org.br/tcc_get.php?cpf=82658773587\&d=20191117204313\&h=0ac694e0b4b4854 a099341280f55e55c9c1e6e2b Acesso em 08 de out. de 2019.

BRACKMANN, Christian P.; BOUCINHA, Rafael M.; Román-González, Marcos; BARONE, Dante; CASALI, Ana. Pensamento Computacional Desplugado: Ensino e Avaliação na Educação Primária Espanhola. Anais dos Workshops do Congresso Brasileiro de Informática na Educação. p. 982-991, 2017. 
Edição Especial: XVI Encontro sobre Investigação na Escola - EIE

ISSN: $2595-4520$

Vol. 4, n. 2. 2021

BRASIL. Base Nacional Comum Curricular: Educação Infantil e Ensino

Fundamental. Brasília: MEC/Secretaria de Educação Básica, 2017. Disponível em: <http://basenacionalcomum.mec.gov.br/abase/<. Acesso em 01 de dez. de 2020.

MEC. Instituto Nacional de Estudos e Pesquisas Educacionais (Inep).

Relatório Brasil no Pisa 2018. Brasília: Inep, 2020. Disponível em:

<http://portal.inep.gov.br/documents/186968/484421/RELAT\%C3\%93RIO+BRASIL+ NO+PISA+2018/3e89677c-221c-4fa4-91ee-08a48818604c?version=1.0<. Acesso em:

01 dez. 2020.

MEC. Instituto Nacional de Estudos e Pesquisas Educacionais (Inep).

Sistema de Avaliação da Educação Básica (SAEB) : Evidências da Edição 2017.

Brasília: Inep, 2020. Disponível em: <http://portal.inep.gov.br/artigo/-

/asset_publisher/B4AQV9zFY7Bv/content/saeb-2017-revela-que-apenas-1-6-dosestudantes-brasileiros-do-ensino-medio-demonstraram-niveis-de-aprendizagemconsiderados-adequados-em-lingua-portug/21206<. Acesso em: 01 dez. 2020.

FELBER, Denise; KRAUSE, João Carlos; VENQUIARUTO, Luciana Dornelles. O uso de jogos digitais como ferramenta de auxílio para o ensino de Física. Revista Insignare Scientia - RIS, v.1, n.2, p.1-25, 23 ago. 2018.

FREIRE, Paulo. Pedagogia da Autonomia: saberes necessários à prática educativa. São Paulo: Paz e Terra, 1996.

WING, Jeannette. Pensamento Computacional: Um conjunto de atitudes e habilidades que todos, não só cientistas da computação, ficaram ansiosos p. Revista Brasileira de Ensino de Ciência e Tecnologia, Ponta Grossa, v. 9, n. 2, p.1-10, ago. 2016.

Quadrimestral. Tradução de Cleverson Sebastião dos Anjos: IF-PR. Disponível em: <https://periodicos.utfpr.edu.br/rbect/article/view/4711, <. Acesso em: 25 nov. 2019.

Recebido em: $30 / 04 / 2020$

Aceito em: 19/11/2020 